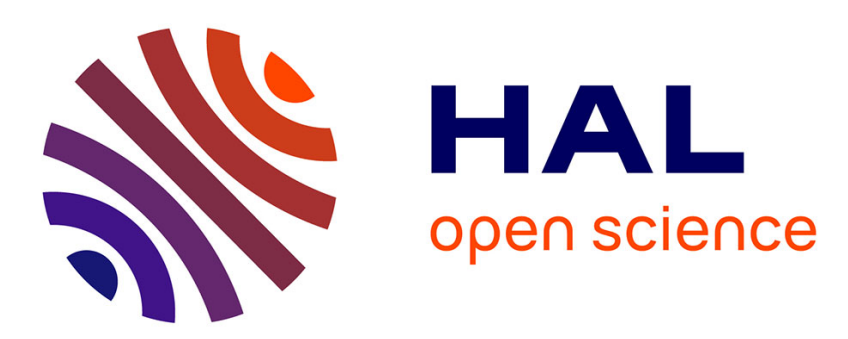

\title{
Les lois de l'aimantation et de la subdivision en domaines élémentaires d'un monocristal de fer \\ Louis Néel
}

\section{To cite this version:}

Louis Néel. Les lois de l'aimantation et de la subdivision en domaines élémentaires d'un monocristal de fer. J. Phys. Radium, 1944, 5 (11), pp.241-251. 10.1051/jphysrad:01944005011024100 . jpa00233889

\section{HAL Id: jpa-00233889 https://hal.science/jpa-00233889}

Submitted on 1 Jan 1944

HAL is a multi-disciplinary open access archive for the deposit and dissemination of scientific research documents, whether they are published or not. The documents may come from teaching and research institutions in France or abroad, or from public or private research centers.
L'archive ouverte pluridisciplinaire HAL, est destinée au dépôt et à la diffusion de documents scientifiques de niveau recherche, publiés ou non, émanant des établissements d'enseignement et de recherche français ou étrangers, des laboratoires publics ou privés. 


\title{
LE JOURNAL DE PHYSIQUE
}

\section{LES LOIS DE L'AIMANTATION ET DE LA SUBDIVISION EN DOMAINES ÉLÉMENTAIRES D'UN MONOGRISTAL DE FER}

\author{
Par M. Louis NÉEL.
}

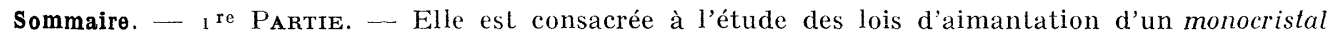
de fer, pur et non déformé, en supposant, à titre d'hypothèse fondamentale, qu'il n'y a pas de charges magnétiques intérieures. Après avoir partagé les domaines élémentaircs, au sens de Weiss, en phases correspondant chacune à une direction bien déterminée de l'aimantation spontanée, on montrè, qu'en général, la courbe d'aimantation d'un monocristal de fer se compose de quatre parties successives correspondant chacune à un mode d'aimantation différent. Ces quatre modes, I, II, III et IV, correspondent respectivement à $6,3,2$ et i phases en équilibre.

Dans le mode I, la substance est magnétiquement isotrope, le champ intérieur est nul et l'aimantation ne dépend que de la forme géométrique. Dans le mode II, le champ intérieur est toujours orienté suivant un axe ternaire et les aimantations spontanées des trois phases sont également inclinées sur lui. On trouve, de même, des conditions simples auxquelles satisfont les directions du champ intérieur et des aimantations spontanées des deux phases du mode III.

On étudie ensuite, en détail, les exemples les plus importants au point de vue expérimental. On établit, pour la première fois et l'on compare aux résultats expérimentaux de Kaya et de Sizoo, les lois d'aimantation, selon le mode II, de barreaux minces, orientés d'une manière quelconque, et, selon le mode III, de barreaux dont le grand axe est à peu près perpendiculaire à un axe quaternaire du cristal. On établit également, pour la première fois, les lois d'aimantation selon les modes I, III et IV de disques taillés dans l'un des plans de base du cube et on les compare aux résultats de Webster et de Honda.

Les deux dernières parties sont consacrées à l'étude de la forme et des dimensions des domaines élémentaires qui ont pu être précisées ici pour la première fois.

${ }_{2}$ C P.rrie. - On y montre que les domaines élémentaires ont la forme de feuillets plans, empilés les uns sur les autres, dont le plan est perpendiculaire à un axe ternaire dans le mode II et à un axe binaire dans le mode III. On interprète ainsi les résultats expérimentaux de Sixtus et de Kaya, relatifs à l'orientation des lignes de poudres (diagrammes de Bitter).

3 Partie. - On y établit la théorie de la structure secondaire superficielle (notion des domaines de fermeture) des domaines élémentaires et on l'utilise pour calculer l'épaisseur des domaines en feuillets qui constituent la structure principale. On étudie, dans un cas simple, les variations de l'équidistance des lignes de poudres en fonction du champ appliqué et l'on donne ainsi l'interprétation quantitative des résultats expérimentaux de Sixtus et de Kaya.
\end{abstract}

\section{Introduction. .}

1. Domaines élémentaires et lois d'aimantation. - Après avoir édifié la théorie du ferromagnétisme qui subsiste encore aujourd'hui dans ses grandes lignes, P. Weiss fut obligé de concilier la facilité apparente de la désaimantation et la grandeur du champ moléculaire : il supposa donc qu'un ferromagnétique apparemment désaimanté est, en réalité, subdivisé en domaines élémentaires aimantés individuellement à saturation, mais dans des directions différentes. Cette hypothèse, universellement admise aujourd'hui, fut accueillie au début avec beaucoup de réticences, mais les expériences de Barkhausen, qui mettent en évidence des discontinuités d'aimantation au cours de la description d'un cycle d'hystérésis, furent considérées comme des preuves directes de l'existence des domaines. En réalité, ces discontinuités, tout au moins dans le domaine des aimantations et des champs faibles, ne se rapportent qu'à des déplacements irréversibles des parois de séparation entre domaines contigus qui n'affectent qu'une fraction relativement faible des domaines en cause. Mais beaucoup d'autres 
preuves, moins directes mais plus convaincantes, donnent aujourd'hui aux domaines élémentaires une existence tout à fait certaine.

Un certain nombre de points sont maintenant précisés : on sait notamment que, dans les champs faibles, l'aimantation spontanée ne peut s'orienter que suivant un nombre fini de directions, dites directions privilégiées ou de facile aimantation qui, pour le fer, sont les trois axes quaternaires du cristal, et l'on connaît l'expression de l'énergie, dite énergie magnétocristalline, à dépenser, pour amener l'aimantation spontanée, depuis une direction privilégiée, jusqu'à une direction quelconque : c'est ainsi que, pour l'amener suivant un axe ternaire, il faut dépenser environ $1,4.10^{5} \mathrm{ergs} / \mathrm{cm}^{3}$.

On sait également, depuis les travaux de Bloch [1], que la paroi de séparation entre deux domaines élémentaires est caractérisée par une certaine énergie superficielle et qu'elle possède une épaisseur finie résultant de l'équilibre entre les forces d'échange de Heisenberg qui tendent d'une part à la rendre aussi épaisse que possible et l'énergie magnétocristalline qui tend, d'autre part, à l'amincir. Pour le fer $\left(^{1}\right)$, l'épaisseur de paroi varie entre 5oo et 2 ooo $\AA$ et l'énergie superficielle correspondante entre 0,7 et $\mathrm{i}, 4 \mathrm{ergs} / \mathrm{cm}^{2}$.

On sait aussi, d'autre part, que les changements d'aimantation d'une substance proviennent de deux mécanismes différents : $a$. une rotation, généralement réversible, de l'aimantation spontanée à l'intérieur de chaque domaine, les frontières de celui-ci restant fixes; $b$. un déplacement, réversible ou irréversible, des parois de séparation entre deux domaines contigus dont les directions d'aimantation restent fixes $\left(^{2}\right)$.

Dans le cas général où l'aimantation est quelconque, on est, par contre, bien peu renseigné sur les directions de l'aimantation spontanée à l'intérieur des domaines, sur les différentes espèces possibles de domaines, leurs proportions respectives, leurs formes, leurs dimensions et leurs dispositions relatives dans l'espace.

$\mathrm{Au}$ seul point de vue macroscopique des lois d'aimantation, deux cas seulement ont été traités avec succès : celui d'un monocristal soumis à un champ dirigé suivant un axe de symétrie (axe quaternaire, ternaire ou binaire) et celui d'un cristal soumis à un champ intense. Dans ces deux cas, on ne vérifie, en somme, que la validité de l'expression mathématique de l'énergie magnétocristalline en fonction des cosinus directeurs de l'aimantation

( $\left.{ }^{1}\right)$ Se reporter à ce sujet à un mémoire de l'auteur consacré à une étude détaillée des parois du fer (Cahiers de Physique, à paraitre en I 945). Ce mémoire, auquel il sera plusieurs fois fait allusion dans la suite, sera désigné par la lettre A.

$\left(^{2}\right)$ Voir les expériences d'Elmore (Phys. Rev., ig38, 53 p. 757 ) qui a directement observé au microscope les déplacements discontinus de parois et à la théorie détaillée des mouvements des parois dans les champs faibles établie par Néel (Cahiers de Physique, 1942, no 1 2, p. I et 1943, no 13 , p. I 8 ). et l'on néglige complètement les phénomènes de subdivision en domaines élémentaires. Dans le premier cas, cette omission n'a pas de répercussions graves puisque, par symétrie, les différentes catégories de domaines possédant les mêmes propriétés, tout se passe donc comme s'il n'y avait qu'une seule catégorie de domaines. Il en est de même dans le second cas, car il n'y a alors, réellement, qu'une seule catégorie de domaines par suite de l'intensité du champ appliqué.

Il ressort ainsi de ce bref résumé qu'il n'existe aucune théorie de l'aimantation d'un ferromagnétique qui tienne effectivement compte de la subdivision en domaines élémentaires en en expliquant les différentes modalités.

Nous allons. dans ce mémoire, essayer de jeter les premières bases d'une telle théorie sur des considérations purement énergétiques, en remarquant que, dans cette subdivision en domaines élémentaires, le champ démagnétisant joue un rôle fondamental dont seuls quelques auteurs, de Waard [2], Landau et Lifshitz [3], Gorter [4], entre autres, ont reconnu l'importance. Comme cette subdivision n'est pas en rapport direct avec les phénomènes d'hystérésis, il est tout indiqué de l'étudier sur un monocristal de fer idéalement pur et non déformé. Il se trouve heureusement que les cristaux réels étudiés jusqu'ici par différents expérimentateurs se rapprochent suffisamment de cet état idéal pour se prêter à des vérifications fructueuses de la théorie. Le fer constitue, à cet égard, une substance de choix bien plus intéressante que le nickel dans lequel les tensions internes accidentelles provoquent de graves perturbations par suite de la grandeur de la magnétostriction.

Nous consacrerons la première partie de ce travail à une étude purement formelle de la décomposition en domaines au cours de laquelle nous déterminerons le nombre des catégories distinctes de domaines élémentaires, leurs proportions relatives ainsi que les directions correspondantes de l'aimantation spontanée. Dans une deuxième partie, nous étudierons la forme et l'orientation des domaines. Enfin, dans la troisième partie, nous déterminerons leurs dimensions absolues en liaison avec certains phénomènes compliqués de surface dans lesquels interviennent des domaines spéciaux dits de fermeture.

\section{PREMIÈRE PARTIE.}

\section{Différents modes d'aimantation d'un monocristal.}

2. L'énergie magnétique et les charges intérieures. - Soit une substance ferromagnétique quelconque subdivisée en domaines élémentaires à une échelle suffisamment petite pour qu'on puisse 
la décomposer en éléments de volume $\mathrm{d} \tau$ qui, tout en étant grands vis-à-vis des domaines élémentaires, soient encore assez petits vis-à-vis des dimensions du cristal. Le champ intérieur moyen $\vec{H}_{i}$ et l'aimantation moyenne $\overrightarrow{\mathfrak{J}}$ en un point sont alors définis comme le champ moyen et l'aimantation moyenne d'un élément de volume $\mathrm{d} \tau$ entourant ce point. Le champ moyen $\vec{H}_{i}$ est la somme du champ imposé $\vec{H}$ et d'un champ démagnétisant moyen $\overrightarrow{H_{d}}$ qui est créé par une répartition fictive de charges définie par la densité moyenne en volume $\rho=-\operatorname{div} \vec{\jmath}$ et la densité superficielle moyenne $\vec{\Im}_{N}$.

En un point pris à l'intérieur de l'élément $\mathrm{d} \tau$, l'aimantation locale est égale à $\vec{y}+\vec{i}$ et le champ local est égal à $\vec{H}+\overrightarrow{H_{c l}}+\vec{h}$ : le champ $\vec{h}$ est créé par les charges réparties sur les surfaces de séparation des domaines élémentaires. Les valeurs moyennes de $\vec{h}$ et de $\vec{i}$, dans l'élément $\mathrm{d} \tau$, sont nulles par définition

$$
\int_{\mathrm{d} \tau} \vec{h} \mathrm{~d} v=0, \quad \int_{\mathrm{d} \tau} \vec{i} \mathrm{~d} v=0 .
$$

L'énergie magnétique $W_{M}$ du système est donnée par l'intégrale

$$
W_{M}=-\int \vec{H}(\vec{\jmath}+\vec{i}) \mathrm{d} v+\int \frac{\left(\overrightarrow{\boldsymbol{H}_{d}}+\vec{h}\right)^{2}}{8 \pi} \mathrm{d} v
$$

étendue à tout l'espace.

En décomposant l'espace d'intégration en éléments de volume $\mathrm{d} \tau$ pour lesquels on peut écrire :

$$
\begin{aligned}
\int_{\mathrm{d} \tau} \vec{H} \vec{i} \mathrm{~d} v & =\vec{H} \quad \int_{\mathrm{d} \tau} \vec{i} \mathrm{~d} v=0, \\
\text { et } & \int_{\mathrm{d} \tau} \overrightarrow{\boldsymbol{H}}_{d} \overrightarrow{\boldsymbol{h}} \mathrm{d} v=\overrightarrow{H_{d}} \int_{\mathrm{d} \tau} \vec{h} \mathrm{~d} v=0,
\end{aligned}
$$

on aboutit finalement à l'expression

$$
W_{M}=-\int \vec{H} \vec{\jmath} \mathrm{d} v+\int \frac{H_{d}^{2}}{8 \pi} \mathrm{d} v+\int \frac{h^{2}}{8 \pi} \mathrm{d} v
$$

La présence de charges superficielles sur les parois des domaines introduit donc, dans l'énergie, un terme supplémentaire toujours positif : $\frac{h^{2}}{8 \pi}$ qui peut atteindre des valeurs considérables : $h$ peut, en effet, prendre la valeur $4 \pi J_{s}$ ce qui donne, pour le fer, I,8. Io $\mathrm{ergs}^{7} / \mathrm{cm}^{3}$. Pour bien apprécier cette valeur, il convient de préciser que l'énergie totale de certaines subdivisions que nous aurons l'occasion d'étudier plus loin ne dépasse guère $100 \mathrm{ergs} / \mathrm{cm}^{3}$.

Une subdivision en domaines élémentaires ne comportant pas de charges intérieures libres possède donc une énergie potentielle particulièrement faible, puisque, $h$ étant nul en tout point, le terme supplémentaire de l'énergie est également nul.
En outre, si l'aimantation spontanée des domaines qui constituent une subdivision sans charges intérieures est en équilibre sous l'action du champ appliqué, du champ démagnétisant et des forces magnétocristallines et si les différents domaines sont en équilibre les uns vis-à-vis des autres, la subdivision en question sera stable vis-à-vis de tous les paramètres qui la caractérisent : proportions relatives des domaines, direction des aimantations spontanées et orientation des parois. Nous supposerons qu'elle correspond à l'état le plus stable du cristal sous l'action du champ considéré.

3. Objet de cette étude et hypothèses fondamentales. - Nous nous proposons d'établir les lois d'aimantation d'un monocristal de fer, idéal et parfait, limité par une surface du second degré $\left(^{3}\right)$ et placé dans un champ magnétique uniforme $\vec{H}$. Sous le nom de cristal idéal et parfait, nous entendons un cristal dont le réseau est identique au réseau théorique, sans lacunes, inclusions ni déformations.

A. Conformément aux conclusions du paragraphe précédent, nous admettrons que la structure des domaines élémentaires, suivant lesquels le cristal est décomposé, est telle que les charges intérieures soient nulles partout. Nous montrerons, dans la deuxième partie de ce mémoire qu'il est toujours possible de trouver de telles structures quand il s'agit d'un monocristal (ce ne serait pas possible dans le cas d'un polycristal) et nous en ferons alors une étude détaillée. Pour l'instant, il nous suffira d'admettre leur existence.

B. Puisqu'il n'y a pas de charges intérieures, le champ intérieur $\vec{H}_{i}$ est uniforme et partout égal à la somme vectorielle du champ appliqué $\vec{H}$ et d'un champ démagnétisant uniforme $\vec{H}_{d}$ identique à celui qui régnerait dans le cristal si l'aimantation était uniforme et égale à l'aimantation moyenne.

C. En outre, nous admettrons qu'il est possible de décomposer le cristal en un nombre fini de phases possédant chacune une aimantation uniforme. Une phase $i$ comprend ainsi tous les domaines dont l'aimantation spontanée $\overrightarrow{\breve{J}}_{i}$ est parallèle à une certaine direction. En module, cette aimantation spontanée est naturellement égale à l'aimantation à saturation à la température considérée, soit $\overrightarrow{\mathscr{J}}_{s}=\mathrm{I} 710 \mathrm{cgs}$, à la température ordinaire. Si nous désignons par $x_{\imath}$

$\left({ }^{3}\right)$ On sait qu'un corps, uniformément aimanté et limité par une surface du second degré, possède un champ démagnétisant uniforme : placé dans un champ uniforme, il prend donc une aimantation uniforme; il en est de même lorsque le champ démagnétisant est négligeable, c'est-à-dire lorsque la substance, isotrope, est taillée en forme de cylindre très allongé de section quelconque et cst aimanté suivant la direction des génératrices. 
la fraction du volume total occupée par la phase $i$, on aura, par définition :

$$
\sum x_{i}=1, \quad \sum x_{i} \vec{J}_{i}=\vec{J} .
$$

D. Enfin, nous admettrons qu'il existe un mécanisme réversible, en fait le déplacement des parois, permettant d'accrô̂tre une phase quelconque aux dépens d'une autre phase quelconque et nous étudierons ainsi les équilibres entre les phases. Nous ne tiendrons compte, dans cette première partie, que de l'énergie magnétique et de l'énergie magnétocristalline : nous négligerons l'énergie superficielle des parois de séparation entre les domaines.

4. Les positions d'équilibre de l'aimantation spontanée dans un champ donné. - Un premier. problème se pose : dénombrer les phases possibles La remarque suivante en facilite la solution : dans l'état d'équilibre, l'aimantation spontanée $\vec{\Im}_{i}$ de chacune des phases est en équilibre sous l'action combinée du champ intérieur $\vec{H}_{i}$ et des forces magnétocristallines de couplage avec le réseau cristallin. Il importe donc, en premier lieu, d'étudier cet équilibre.

Dans les cristaux ferromagnétiques du système cubique, on met sous la forme suivante, avec une approximation suffisante dans la plupart des cas, le terme d'énergie potentielle qui correspond aux forces magnétocristallines :

$$
W_{C}=K\left(\alpha^{2} \gamma^{2}+\beta^{2} \gamma^{2}+\gamma^{2} \alpha^{2}\right),
$$

où $\alpha$, $\beta$ et $\gamma$ désignent les cosinus directeurs de l'aimantation spontanée, rapportée aux axes quaternaires du cristal comme axes de coordonnées. Dans le cas du fer, $K$ est positif et égal à $4,3 . \mathrm{IO}^{5} \mathrm{ergs} / \mathrm{cm}^{3}$. Lorsque le champ $\vec{H}_{i}$ est nul, il y a six positions d'équilibre distinctes :

$$
\begin{gathered}
\alpha= \pm 1, \quad \beta=\gamma=0 ; \quad \beta= \pm r, \quad \alpha=\gamma=0 ; \\
\gamma= \pm r, \quad \alpha=\beta=0 .
\end{gathered}
$$

Ces six positions correspondent à une même énergie totale égale à zéro : elles sont donc rigoureusement équivalentes. Ainsi, lorsque le champ intérieur est nul, six phases différentes peuvent subsister en équilibre dans le cristal, en présence les unes des autres.

Appliquons maintenant un champ $H_{i}$, de direction définie par les cosinus directeurs $p, q, r$ rapportés aux axes quaternaires $\mathrm{OX}, \mathrm{OY}, \mathrm{OZ}$, du cristal $(p \geqq q \geqq r)$. Si $H_{i}$ est suffisamment faible, les aimantations spontanées sont peu déviées de leurs positions initiales d'équilibre et les énergies potentielles correspondantes, prennent les valeurs suivantes, au second ordre près :

$$
\mp p H_{i} \mathcal{J}_{s}, \quad \mp q H_{i} \mathcal{J}_{s}, \mp r H_{i} \mathcal{J}_{s} .
$$

Les six positions ainsi obtenues ne sont généra- lement plus équivalentes : la position la plus stable correspond à l'énergie la plus basse, dérivant de la position initiale $\mathrm{OX}$.

En résumé, lorsque $p$ est plus grand que $q$, l'une des phases est énergétiquement plus favorisée que les autres : celle qui correspond à la position initiale OX. la plus rapprochée de la direction positive du champ. Nous admcttrons que l'exactitude de cette proposition, d'ailleurs assez évidente, se conserve pour une valeur quelconque du champ $H_{i}$.

De la proposition précédente, nous déduisons immédiatement que, si le champ intérieur n'est pas nul et si sa direction est telle que $p$ soit plus grand que $q$ et que $r$. les équilibres entre phases sont impossibles : le cristal ne renferme qu'une seule phase.

Par contre, lorsque les cosinus directeurs sont tels que $p=q>r$. il existe deux positions équivalentes d'équilibre, symétriques par rapport au plan $x+y=0$ et, enfin, lorsque $p=q=r$, il existe trois positions d'équilibre équivalentes, symétriques par rapport à l'axe ternaire. Ainsi, pour qu'il y ait équilibre entre trois phases, il faut que le champ intérieur soit parallèle à un axe ternaire; pour qu'il y ait équilibre entre deux phases, sa direction doit être telle que deux des còsinus directeurs soient égaux, le troisième étant plus petit en module que les deux autres.

Ces simples remarques permettent de distinguer quatre modes possibles d'aimantation, suivant le nombre des phases en présence et la nature du champ intérieur. Le Tableau I en résume les caractéristiques. Nous montrerons dans la suite, qu'en général, la courbe d'aimantation d'un monocristal se subdivise en quatre régions, se raccordant à des

\begin{tabular}{|c|c|c|c|}
\hline \multirow{2}{*}{$\begin{array}{c}\text { Mode } \\
\text { d'aimantation. }\end{array}$} & \multicolumn{2}{|c|}{ Champ intérieur. } & \multirow{2}{*}{$\begin{array}{c}\text { Nombre } \\
\text { des phases. }\end{array}$} \\
\hline & Intensití. & Direction. & \\
\hline I $\ldots \ldots \ldots$ & $H_{i}=0$ & & 6 \\
\hline II $\ldots \ldots \ldots$ & $H_{i}>0$ & $p=q=r$ & 3 \\
\hline III ..... & $I_{i}>0$ & $p=q>r$ & 2 \\
\hline IV ...... & $H_{i}>0$ & $p>q>r$ & I \\
\hline
\end{tabular}
points anguleux, correspondant chacune à un mode d'aimantation différent.

Tableau 1. - Les différents modes d'aimantation d'un monocristal idéal de fer.

כ. Représentation géométrique de l'aimantation moyenne. - Plaçons-nous dans le cas où il y a six phases en présence (mode I) et désignons par $x$ et $\bar{x}$ les fractions du volume total occupées par les phases respectivement aimantées suivant OX et la direction inverse $O X^{\prime}$. Désignons de même, par $y, \bar{y}$ et $z, \bar{z}$ les fractions occupées par les phases aimantées suivant $\mathrm{OY}, \mathrm{OY}^{\prime}$ et $\mathrm{OZ}, \mathrm{OZ}^{\prime}$. Par défi- 
nition,

$$
x+\bar{x}+y+\bar{y}+z+\bar{z}=1,
$$

tandis que l'aimantation moyenne $\vec{\jmath}$ s'écrit en fonction des vecteurs unitaires $\vec{i}, \vec{j}, \vec{k}$ portés par les axes quaternaires :

$$
\vec{J}=[(x-\bar{x}) \vec{i}+(y-\bar{y}) \vec{j}+(z-z) \vec{k}] J_{s} .
$$

Menons, par un point $\mathrm{O}$, trois axes rectangulaires $\mathrm{OX}$, $\mathrm{OY}, \mathrm{OZ}$, parallèles aux axes quaternaires du cristal et un verteur $\overline{\mathrm{OM}}$ égal à l'aimantation moyenne. Il est visible que le point $\mathrm{M}$ sera toujours à l'intérieur de l'octaèdre régulier $\mathrm{AA}^{\prime} \mathrm{BB}^{\prime} \mathrm{CC}^{\prime}$ limité par les huit plans $\pm x^{\prime} \pm z^{\prime} \pm y^{\prime}=\mathfrak{y}_{;}(f i g . \quad 1)$. Cet octaèdre est inscrit à l'intérieur de la sphère de centre $\mathrm{O}$ et de rayon $\mathfrak{J}_{s}$. Réciproquement, tout point $\mathrm{M}$ pris à l'intérieur de cet octaèdre représente un état possible d'aimantation selon le mode I.

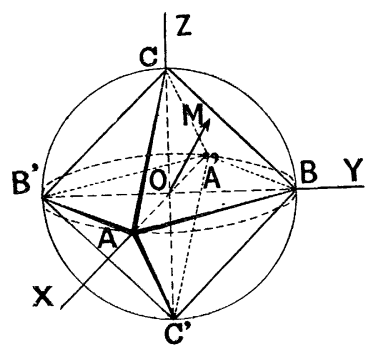

Fig. I.

De même, une étude géométrique élémentaire montre que tous les points représentatifs des états d'aimantation appartenant aux modes II et III sont situés dans le volume qui est compris entre l'octaèdre et la sphère circonscrite. Les points représentatifs du mode IV sont situés à la surface de cette sphère. Le point représentatif de l'aimantation d'un corps désaimanté est en O. Lorsqu'on aimantera ce corps à saturation, le point représentatif $M$ passera donc du point $O$ jusqu'à la surface de la sphère de rayon $\mathcal{J}_{s}$. Il traversera l'octaèdre : la phase initiale de l'aimantation se fera donc suivant le mode I.

6. La phase initiale de l'aimantation (mode I). - Comme dans ce mode d'aimantation, le champ intérieur est nul, l'aimantation prise par le cristal, placé dans un champ imposé $\vec{H}$, se déterminera en écrivant que le champ démagnétisant de forme $\overrightarrow{H_{l}}$ est égal et opposé au champ imposé $\vec{H}$. Pour ne pas compliquer l'écriture, nous supposerons désormais, dans tout ce qui va suivre, le champ imposé toujours parallèle à l'un des trois axes principaux de l'ellipsoüde qui limite le cristal. Malgré cette restriction, nous engloberons néanmoins ainsi toutes les expériences relatives aux monocristaux, tandis que le cas général, par contre, ne nous apporterait rien d'essentiellement nouveau. Dans ces conditions, puisque l'aimantation est dirigée suivant un axe principal, le champ démagnétisant est également dirigé suivant cet axe et égal à $-\mathrm{N} \mathcal{J}$ en désignant par $N$ le coefficient de champ démagnétisant relatif à l'axe principal correspondant. L'aimantation moyenne est donc parallèle et proportionnelle au champ imposé :

$$
\vec{J}=\frac{\mathrm{I}}{N} \vec{H}
$$

Elle est indépendante de l'orientation des axes $d u$ cristal par rapport aux axes de l'ellipsoüde. L'expérience montre d'ailleurs [j] que les cristaux cubiques sont magnétiquement isotropes dans les champs faibles.

La proportionnalité de l'aimantation au champ, définie par la loi (5), se poursuit aussi longtemps que l'extrémité $M$ du vecteur $\overline{O M}$ reste à l'intérieur de l'octaèdre. La traversée de la surface de l'octaèdre est accompagnée d'un changement dans le mode d'aimantation dû à ce que le champ intérieur devient différent de zéro. Nous donnerons le nom d'aimantation rémanente idéale à l'aimantation $\mathscr{J}_{r}$ correspondant à cette transition. Nous en obtiendrons la valeur en prenant l'intersection de la face $x^{\prime}+y^{\prime}+z^{\prime}=\mathscr{J}_{s}$ de l'octaèdre avec une droite passant par $\mathrm{O}$, parallèle au champ appliqué. Soient $l, m, n$ les cosinus directeurs du champ et $x \mathscr{J}_{s}, y \mathscr{J}_{s}, \quad z \mathfrak{J}_{;}$les coordonnées du point d'intersection, on aura

$$
\begin{aligned}
\frac{x \mathscr{J}_{s}}{l} & =\frac{y \mathcal{J}_{s}}{m}=\frac{z J_{s}}{n} \\
& =\frac{\mathfrak{J}_{s}}{l+m+n}=\sqrt{\frac{\left(x^{2}+y^{2}+z^{2}\right) \mathfrak{J}_{s}^{2}}{l^{2}+m^{2}+n^{2}}}=\mathfrak{J}_{r} .
\end{aligned}
$$

L'aimantation rémanente idéale est donc liée aux cosinus directeurs du champ appliqué par la relation

$$
\mathfrak{J}_{r}=\frac{\mathscr{J}_{s}}{l+m+n} .
$$

Kaya [6], à titre purement empirique, a proposé, le premier, cette formule pour représenter le résultat de ses expériences sur les barreaux monocristallins de fer, mais la signification théorique n'en fut dégagée que plus tard par Gorter [7].

L'aimantation donnée par la formule (5) est bien déterminée; néanmoins, les proportions relatives des six phases en présence ne sont pas déterminées puisque les six quantités $x, \bar{x}, y, \bar{y}, z, \bar{z}$ ne sont données que par quatre relations : la relation (3) et les trois relations déduites de l'équation vectorielle (4). Mais la situation change lorsqu'on atteint l'aimantation rémanente : à ce moment, les proportions $\bar{x}, \bar{y}, \bar{z}$ des phases aimantées suivant $\mathrm{OX}^{\prime}$, $\mathrm{OY}^{\prime}, \mathrm{OZ}^{\prime}$ se réduisent à zéro et il ne reste plus que 
trois phases aimantées suivant OX, OY, OZ, dont les proportions sont respectivement égales, d'après (4) et (6) à

$$
\left.\begin{array}{c}
x=\frac{l}{l+m+n}, \quad y=\frac{m}{l+m+n}, \\
z=\frac{n}{l+m+n} .
\end{array}\right\}
$$

Si aucun des cosinus directeurs n'est nul, trois phases coexistent alors dans la substance, de sorte que, par continuité, une augmentation subséquente du champ imposé, doit faire croître l'aimantation selon le mode II.

7. Étude de l'aimantation selon le mode II dans le cas des barreaux. - Nous venons de montrer que, dans le cas général, la première région de la courbe d'aimantation, dans laquelle l'aimantation se fait suivant le mode I avec 6 phases, est suivie d'une région dans laquelle l'aimantation doit se faire suivant le mode II avec 3 phases. Étudions maintenant cette deuxième région sur l'exemple particulièrement important au point de vue expérimental de barreaux monocristallins. Ces barreaux sont toujours suffisamment allongés pour que le coefficient de champ démagnétisant transversal soit très voisin de $2 \pi$. Nous supposerons, en outre, pour simplifier l'exposé, que le coefficient de champ démagnétisant longitudinal est nul : pratiquement, il n'en est jamais ainsi, mais il est facile de se ramener à ce cas par une simple correction, en retranchant des valeurs du champ imposé le champ démagnétisant longitudinal $N \mathcal{J}$, toujours faible d'ailleurs.

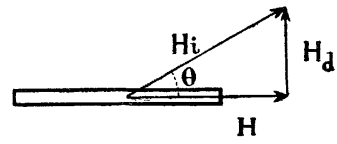

Fig. 2.

Nous désignerons par $l, m, n(l>m>n>0)$, les cosinus directeurs du grand axe du barreau par rapport aux axes quaternaires du cristal, choisis avec des sens positifs convenables. Le champ extérieur sera également parallèle à ce grand axe. L'aimantation rémanente $\mathfrak{J}_{r}=\mathscr{J}_{s}:(l+m+n)$ est atteinte pour un champ infiniment faible au delà duquel l'aimantation se poursuit selon le mode II : le champ intérieur $H_{i}$ doit donc être dirigé suivant l'axe ternaire du cristal le plus rapproché du champ imposé, celui dont les trois cosinus directeurs sont égaux à $\mathrm{I}: \sqrt{3}$. Comme le champ démagnétisant longitudinal est nul, le champ intérieur $\vec{H}_{i}$ se réduit à la somme géométrique du champ extérieur $\vec{H}$, parallèle à l'axe du barreau, et d'un champ démagnétisant transversal $\overrightarrow{H_{d}}$, perpendiculaire à l'axe du barreau (fig. 2). Désignons par 0 l'angle de $\vec{H}$ avec $\vec{H}_{i}$; on a

$$
\cos \theta=(l+m+n): v^{\prime} \overline{3}
$$

d'où

$$
H_{i}=H: \cos \theta=1 \overline{3} H:(l+m+n) .
$$

Sous l'action de ce champ intérieur $\vec{H}_{i}$, les aimantations spontanées $\mathrm{OA}, \mathrm{OB}$, OC des trois phases, primitivement dirigées suivant $\mathrm{OX}, \mathrm{OY}, \mathrm{OZ}$, tournent et se rapprochent symétriquement de l'axe ternaire, en $\mathrm{OA}^{\prime}, \mathrm{OB}^{\prime}, \mathrm{OC}^{\prime}(f i g .3)$. Le plan $\mathrm{A}^{\prime} \mathrm{B}^{\prime} \mathrm{C}^{\prime}$, normal à

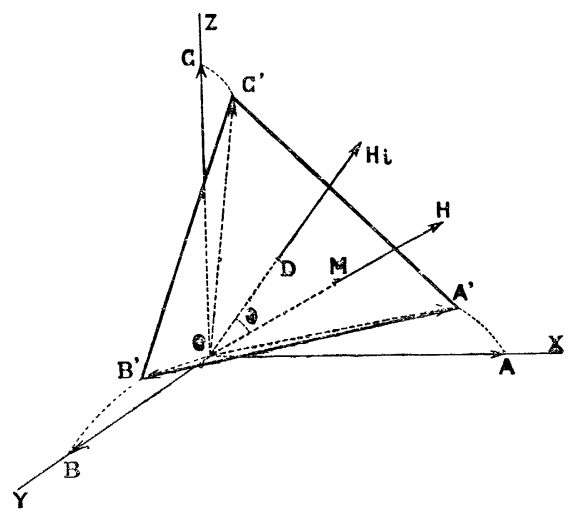

Fig. 3.

l'axe ternaire, coupe cet axe en D et la longueur OD est une fonction de $H_{i}$ :

$$
\mathrm{OD}=F\left(\boldsymbol{H}_{i}\right)
$$

D'autre part, si nous désignons par $\xi$, $n$, $\zeta$, les proportions des phases aimantées suivant $\mathrm{OA}^{\prime}, \mathrm{OB}^{\prime}$ et $\mathrm{OC}^{\prime}(\zeta+\eta+\zeta=\mathrm{I})$ et par $\vec{i}^{\prime}, \vec{j}^{\prime}$ et $\vec{k}^{\prime}$ les vecteurs unitaires correspondants, l'aimantation moyenne du système est alors donnée par

$$
\overrightarrow{\mathrm{OM}}=\left(\xi \overrightarrow{i^{\prime}}+\eta \overrightarrow{j^{\prime}}+\zeta \overrightarrow{k^{\prime}}\right) \mathfrak{J}_{s} .
$$

Le point $\mathrm{M}$ est donc situé dans le plan $\mathrm{A}^{\prime} \mathrm{B}^{\prime} \mathrm{C}^{\prime}$ et à l'intérieur de ce triangle. Mais nous savons aussi que cette aimantation $\mathrm{OM}$ doit être presque exactement parallèle à l'axe du barreau, puisqu'à cause de la grande valeur du coefficient de champ démagnétisant transversal, il suffit d'une inclinaison extrêmement faible de l'aimantation sur le grand axe pour produire le champ démagnétisant transversal nécessaire $\vec{H}_{d}$. Il en résulte que l'aimantation $\vec{J}=\overrightarrow{\mathrm{OM}}$ s'obtiendra avec une approximation suffisante en prenant l'intersection du plan $\mathrm{A}^{\prime} \mathrm{B}^{\prime} \mathrm{C}^{\prime}$ avec une parallèle à l'axe du barreau menée par $\mathrm{O}$. On aura

$$
\mathrm{OM}=\mathrm{OD}: \cos \theta \text {; }
$$


d'où finalement,

$$
\mathfrak{J} \frac{l+m+n}{\sqrt{3}}=F\left(\frac{H \sqrt{3}}{l+m+n}\right) \quad(\text { mode II }) .
$$

Ainsi, la courbe obtenue en représentant $\mathfrak{y}(l+m+n)$ en fonction de $H:(l+m+n)$ est identique pour tous les barreaux quelle que soit leur orientation.

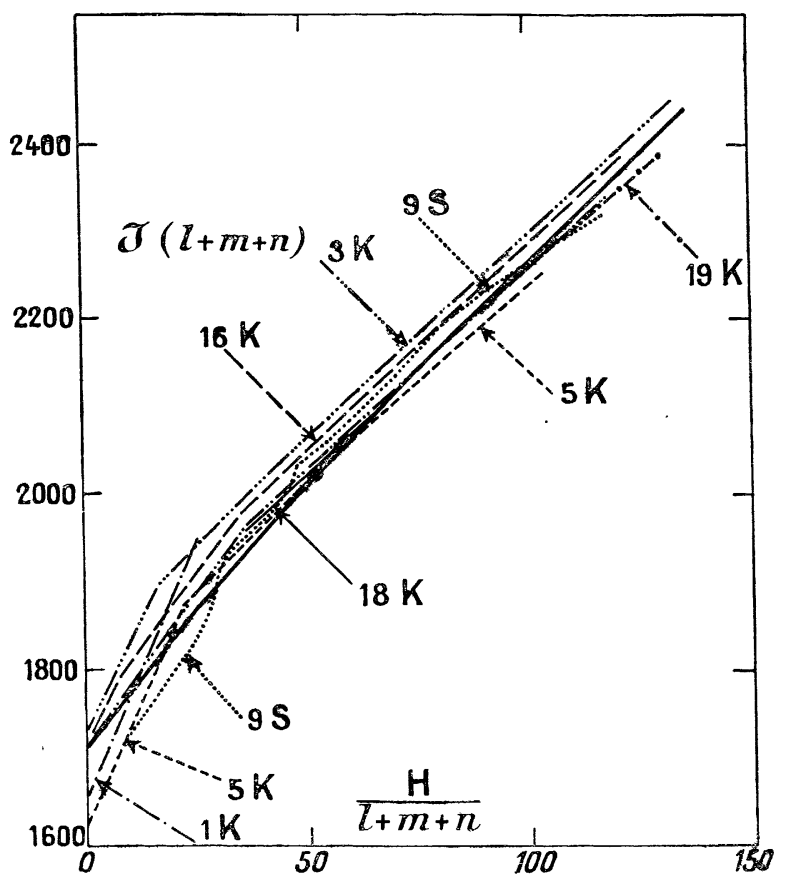

Fig. 4. - Courbes d'aimantation, selon le mode II, de barreaux monocristallins de fer diversement orientés. La courbe en trait plein est la courbe théorique tandis que les courbes expérimentales sont ponctuées. Les numéros suivis d'un $\mathrm{K}$ se rapportent à des barreaux de Kaya; ceux qui sont suivis d'un S à des barreaux de Sizoo.

Cette courbe est d'ailleurs facile à calculer puisque OD est la projection sur un axe ternaire du vecteur $\mathrm{OA}^{\prime}$ : c'est une des positions d'équilibre de l'aimantation spontanée sous l'action combinée des forces magnétocristallines et d'un champ $H_{i}$ parallèle à l'axe ternaire. Notre courbe est donc la courbe classique d'aimantation suivant l'axe ternaire, calculée par Akulov [8]. En posant

on trouve

$$
\eta=\mathrm{OD}: \mathfrak{J}_{s}
$$

$$
\begin{aligned}
3 H_{i} \mathscr{J}_{s}: K= & \left(\eta+\sqrt{2} \sqrt{\mathrm{I}-\eta^{2}}\right) \\
& \times\left(2 \eta \sqrt{2}+\sqrt{\mathrm{I}-\eta^{2}}\right)\left(\eta \sqrt{2}-\sqrt{\mathrm{I}-\eta^{2}}\right),
\end{aligned}
$$

d'où, par inversion, la fonction (9).

Nous avons reporté sur la figure 4, dans la représentation indiquée plus haut, de nombreuses mesures de Kaya [9] et de Sizoo [10] relatives à des barreaux diversement orientés ainsi que la courbe théorique déduite de la relation (12). Les écarts avec la courbe théorique atteignent exceptionnellement 2 pour 100 et paraissent imputables en majeure partie à de faibles erreurs, de l'ordre du degré, sur l'orientation des axes. L'accord général du calcul avec l'expérience est donc très satisfaisant et ne laisse pas de doute sur l'exactitude du mécanisme que nous venons de décrire.

La loi (11) est valable aussi longtemps qu'il reste effectivement trois phases en présence. Or, d'après la relation (10), l'une des phases disparaît lorsque, dans la décomposition du vecteur $\mathrm{OM}$ suivant les trois directions $\mathrm{OA}^{\prime}, \mathrm{OB}^{\prime}, \mathrm{OC}^{\prime}$, l'une des composantes s'annule : c'est précisément ce qui se passe, lorsque, le triangle $\mathrm{A}^{\prime} \mathrm{B}^{\prime} \mathrm{C}^{\prime}$ se rétrécissant sous l'influence du champ $H_{i}$ croissant, la direction $\mathrm{OM}$ se trouve dans le plan $\mathrm{A}^{\prime} \mathrm{OB}^{\prime}$. A partir de ce moment, il n'y a plus que deux phases en présence et le processus d'aimantation se poursuit forcément suivant le mode III. Un calcul élémentaire de trigonométrie sphérique montre que l'aimantation $\mathscr{J}_{c}$ correspondant à cette transition, est donnée par les formules

$$
\mathscr{J}_{c}=\mathscr{J}_{s} \cos \lambda \quad \text { arec } \operatorname{tg} \lambda=\frac{2(l+m)-4 n}{\sqrt{2}(l+m+n)} .
$$

Cette aimantation $J_{c}$, au delà de laquelle la loi (11) n'est plus valable, est variable selon l'orientation du barreau considéré. On a utilisé les relations (13) pour arrêter aux points convenables les courbes de la figure 4 .

8. Aimantation d'un barreau selon le mode III. - Lorsque l'aimantation $\mathfrak{J}_{c}$ est dépassée, il ne subsiste plus que deux phases aimantées suivant les directions $\mathrm{OA}^{\prime}$ et $\mathrm{OB}^{\prime}$ qui correspondent aux deux axes quaternaires OX et OY les plus rapprochés du grand axe du barreau. Le calcul de l'aimantation est alors assez pénible sauf dans le cas où $n=0$ c'est-à-dire lorsque le grand axe du barreau est situé dans l'un des plans de base du cube : d'ailleurs. dans ce cas, le mode II n'existe pas, on passe directement du mode I au mode III. Lorsqu'il en est ainsi, un des plans de symétrie géométrique du barreau est aussi un plan de symétrie magnétique. Le champ appliqué étant dans ce plan, il en est de même de l'aimantation moyenne et du champ intérieur : celui-ci est donc parallèle à l'axe binaire qui bissecte intérieurement les deux axes OX et OY. On a affaire à un problème plan et en calquant exactement les raisonnements sur ceux du problème à trois dimensions étudié dans le paragraphe précédent, on montre que, dans le mode III, si $n$ est nul, l'aimantation est reliée au champ par la formule

$$
\text { · } J \frac{l+m}{\sqrt{2}}=G\left(\frac{H \sqrt{2}}{l+m}\right) \quad(\text { mode III ). }
$$

Cette fois-ci, la relation $\mathfrak{J} G H(=)$ représente 
la courbe classique d'aimantation suivant l'axe

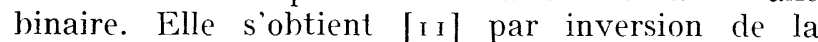
fonction

$$
H_{i} \mathcal{J}=? \boldsymbol{K} r_{1}\left(\cdot r_{1}^{2}-1\right) \text { avec } r_{1}=\mathfrak{J}: \mathcal{J}_{1}
$$

Quand $H$ est petit, le commencement du développement en série de la relation (14) s'écrit

$$
\frac{\mathfrak{J}}{\mathfrak{J}_{s}}=\frac{\mathrm{I}}{l+m}+\frac{\mathfrak{J}_{s} H}{2 K(l+m)^{2}}+\ldots \quad(\text { mode III }) . \quad \text { (I6) }
$$

La tangente initiale a donc pour pente

$$
\mathscr{J}_{5}: 2 K(l+m)^{2} \text {. }
$$

Comme nous le verrons plus loin, la relation (11) est bien vérifiée par l'expérience, mais comme il est extrêmement difficile d'obtenir des barreaux exactement orientés suivant l'un des plans de base du cube, $n$ n'est jamais exactement nul et il est utile d'examiner ce qui se passe dans ce cas.

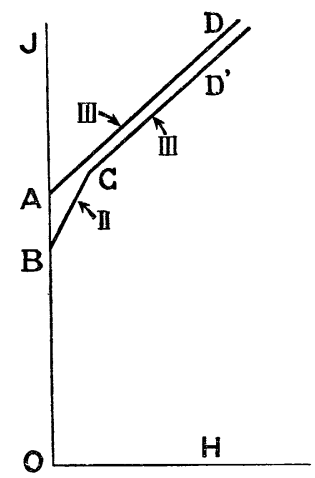

Fig. 5. - AD représente la courbe d'aimantation d'un barreau exactement orienté suivant l'un des plans de base du cube, tandis que si l'orientation est un peu différente, on obtient la courbe $\mathrm{BCD}^{\prime}$ (schématique).

Nous allons donc donner à $n$ une valeur très petite en conservant aux cosinus directeurs $l$ et $m$ la même valeur, au second ordre près en $n$, que dans le cas où $n$ est nul. Dans cette opération, l'aimantation rémanente (fig. 5) baisse de la valeur

à la valeur

$$
\mathrm{OA}=\mathcal{J}_{s}:(l+m)
$$

$$
\mathrm{OB}=\mathcal{J}_{s}:(l+m+n) .
$$

Par contre, comme à partir du point $\mathrm{B}$, l'aimantation se fait suivant le mode II, il faut utiliser la formule (11) dont le développement en série au voisinage de la rémanente s'écrit

$$
\begin{aligned}
\frac{\mathfrak{J}}{\mathfrak{J}_{s}}= & \frac{\mathrm{I}}{l+m+n} \\
& +\frac{\mathfrak{J}_{s} H}{K(l+m+n)^{2}}+\ldots \quad(\text { mode II }) .
\end{aligned}
$$

La pente initiale est alors égale à $\mathcal{J}_{s}: K(l+m+n)^{2}$; sa valeur est sensiblement double de la valeur correspondant à $n=$ o. La pente de BC (fig. 5) est donc double de la pente de AD. D'autre part, comme $n$ est petit, la portion de courbe correspondant au mode II est aussi très petite : il doit donc se produire en $\mathrm{C}$ un coude au delà duquel l'aimantation augmentera suivant le mode III. Nous avons représenté, sur la figure, l'elément $\mathrm{CD}^{\prime}$ très voisin de l'élément $\mathrm{AD}$ : le calcul montre, en effet, que la différence est du second ordre en $n$. On obtient, comme équation de la droite $\mathrm{CD}^{\prime}$,

$$
\begin{aligned}
\frac{\mathfrak{J}}{\mathfrak{J}_{*}} & {\left[\mathrm{I}+\frac{n^{2}}{(l+m)^{2}}\right] } \\
& =\frac{1}{l+m}+\frac{\mathfrak{J}, I I}{? \boldsymbol{K}(l+m)^{2}}+\ldots \quad(n \ll m<\mathrm{I}) .
\end{aligned}
$$

tandis que l'équation de la droite $\mathrm{AD}$ est donnée par (16). Cette remarque est importante car nous voyons ainsi que la loi (14), qui n'est strictement valable que pour $n=0$, représente cependant, avec une précision relative qui est de l'ordre

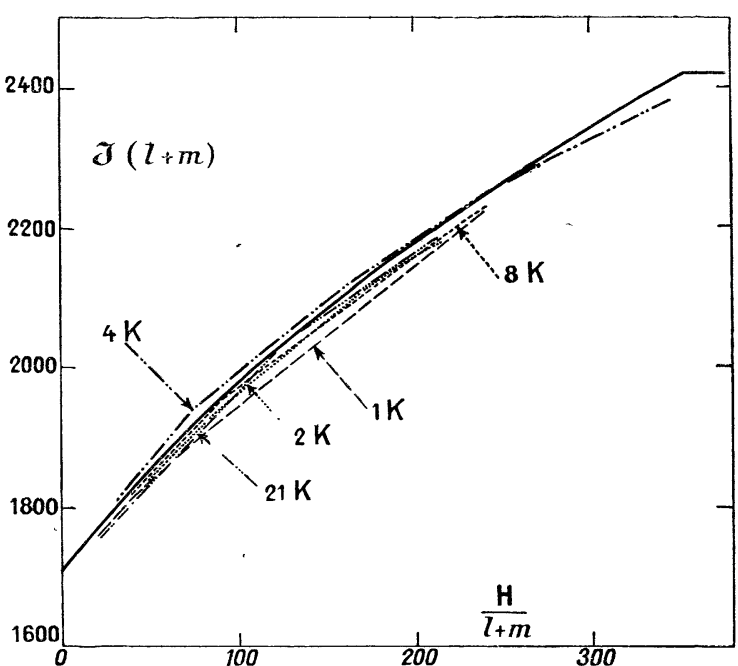

Fig. 6. - Courbes d'aimantation, selon le mode III, de barreaux approximativement orientés suivant l'un des plans de base du cube. La courbe en trait plein est la courbe théorique. Les courbes ponctuées se rapportent à des barreaux étudiés par Kaya et les numéros correspondants à ceux de son mémoire.

de $n^{2}:(l+m)^{2}$, les parties de la courbe d'aimantation, correspondant au mode III, relatives à des barreaux qui ne sont pas exactement orientés suivant l'une des faces du cube. L'erreur commise de ce chef est inférieure à 0,5 pour roo lorsque $n$ est inférieur ou égal à $o, \mathrm{I}$.

Nous avons mis à profit cette remarque pour tracer la figure 6 qui représente les valeurs théoriques de $J(l+m)$ en fonction de $H:(l+m)$, comparées aux valeurs expérimentales de Sizoo et de Kaya (loc. cit.), relatives à une série de barreaux 
d'orientations variées, mais tels que $n$ était inféà $\mathrm{o}, \mathrm{r}$. Nous avons cependant utilisé également un barreau avec $n=0 . \mathrm{I} 3$, mais nous avons alors effectué la correction donnée par la formule (18). Naturellement, nous n'avons tracé que les portions de courbe correspondant au mode III. Comme dans les exemples du paragraphe précédent, l'accord général de la théorie avec l'expérience est extrêmement satisfaisant.

Lorsque le champ extérieur atteint une valeur suffisante, l'une des deux phases se réduit à zéro et tous les domaines ne sont plus aimantés que suivant une seule direction, l'aimantation se faisant désormais suivant le mode IV. Pour compléter notre étude, nous allons étudier le détail de ce phénomène dans un cas important au point de vue expérimental : celui de disques de révolution (ellipsoïdes aplatis) dont le plan équatorial est perpendiculaire à un axe quaternaire du cristal.

9. Aimantation des disques suivant les modes III et IV. - Nous supposerons que le champ extérieur $H$, uniforme, est parallèle au plan équatorial du disque et qu'il fait un angle $\varphi_{\text {avec la }}$ bissectrice OC des deux axes quaternaires OX et OY que contient ce plan (fig. 7).

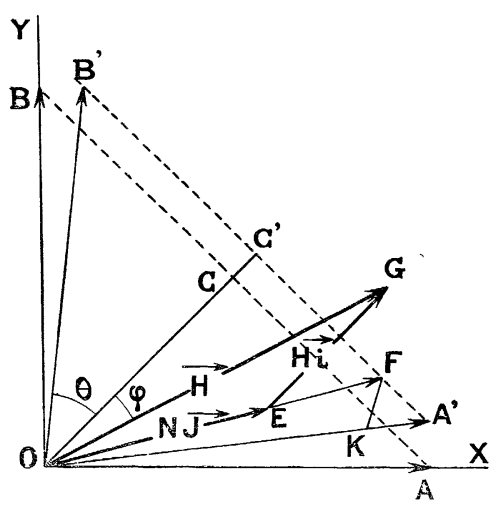

Fig. 7. - Diagramme des aimantations et des champs relatif à un ellipsoïde de révolution aplati dont le petit axe coïncide avec un axe quaternaire du cristal.

Nous savons déjà, d'après les résultats généraux du paragraphe 5, que, dans la phase initiale (mode I), l'aimantation est parallèle et proportionnelle au champ jusqu'à ce qu'elle atteigne la valeur

$$
\mathfrak{J}_{r}=\frac{\mathfrak{J}_{s}}{l+m}=\frac{\mathfrak{J}_{s}}{\sqrt{2} \cos \vartheta_{\imath}} .
$$

Il faut pour cela que le champ extérieur prenne la

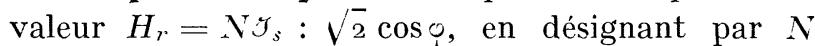
le coefficient de champ démagnétisant du disque dans une direction perpendiculaire au petit axe.
Lorsque $\vec{H}$ dépasse cette valeur, le champ intérieur $\vec{H}_{i}$ n'est plus nul et, puisqu'il s'agit d'un équilibre entre deux phases (mode III), il est dans le plan perpendiculaire à la bissectrice extérieure des deux axes OX et OY. Par raison de symétrie, il est donc dirigé suivant OC. Puisque $\vec{H}$ et $\vec{H}_{i}$ sont situés dans le plan XOY, il résulte de la relation

$$
\vec{H}_{i}=\vec{H}-N \overrightarrow{\mathfrak{J}}
$$

que l'aimantation $\overrightarrow{\mathfrak{g}}$ est également dans ce plan, mais elle n'est pas parallèle au champ appliqué $\vec{H}$ : elle possède une composante $\mathfrak{J}_{N}$, normale au champ, que nous nous proposons en particulier de calculer.

Représentons, sur la figure 7 , les segments

$$
\overline{\mathrm{UE}}=N \vec{\jmath} ; \quad \overline{\mathrm{EG}}=\vec{H}_{i} ; \quad \overline{\mathrm{OG}}=I I .
$$

Sous l'action du champ intérieur $\vec{H}_{i}$, l'aimantation spontanée de chacune des deux phases tourne légèrement de $\mathrm{OA}$ en $\mathrm{OA}^{\prime}$ et de $\mathrm{OB}$ en $\mathrm{OB}^{\prime}$, symétriquement par rapport à $\mathrm{OC}$. Le vecteur $\mathrm{OB}^{\prime}$ est en équilibre sous l'action des forces magnétocristallines et du champ $H_{i}$. En désignant par 0 l'angle de $\mathrm{OB}^{\prime}$ avec OC, on a, d'une part,

$$
\mathrm{OC}^{\prime}=\mathrm{OB}^{\prime} \cos \theta
$$

tandis que, d'autre part, l'énergie totale du système s'écrit

$$
W=\frac{K}{4} \cos ^{2} 20-\mathfrak{J}_{s} H_{i} \cos \theta
$$

Elle est minimum pour $\frac{\partial W}{\partial \theta}=o$, ce qui donne tous calculs faits,

$$
H_{i}=\frac{2 K}{\mathfrak{J}_{s}} \cos 0\left(2 \cos ^{2} 0-\mathrm{I}\right),
$$

relation qui détermine $\theta$ en fonction de $H_{i}$.

D'autre part, soumis au champ $H_{i}$, le disque se subdivise en domaines élémentaires aimantés, les uns suivant $\mathrm{OA}^{\prime}$, les autres suivant $\mathrm{OB}^{\prime}$. Selon les proportions relatives de ces deux catégories, l'extrémité M du vecteur OM, représentatif de l'aimantation moyenne, décrit la droite $\mathrm{A}^{\prime} \mathrm{B}^{\prime}$, comme nous l'avons expliqué plus haut à propos de la formule (10). Mais, par hypothèse, l'aimantation moyenne est dirigée suivant $\mathrm{OE}$. Elle est donc égale à $\mathrm{OF}$, en désignant par $F$ l'intersection de $\mathrm{OE}$ avec $\mathrm{A}^{\prime} \mathrm{B}^{\prime}$. Projetons le contour $\overline{\mathrm{OG}}=\overline{\mathrm{OE}}+\overline{\mathrm{EG}}$ sur OC, nous obtenons la relation

$$
\begin{aligned}
H \cos \varphi & =N \overline{\mathrm{OC}^{\prime}}+H_{i} \\
& =N \mathscr{J}_{s} \cos \theta+\frac{2 K}{J_{s}} \cos \theta\left(2 \cos ^{2} \theta-\mathrm{I}\right),
\end{aligned}
$$

qui relie le champ extérieur $H$ à l'angle $\theta$. Projetons, d'autre part, le même contour sur une perpendicu- 
laire à $\mathrm{OG}$, nous obtenons l'aimantation normale

$$
\mathfrak{J}_{N}=-\frac{\mathrm{I}}{\boldsymbol{N}} \boldsymbol{H}_{i} \sin \varphi=-\frac{2 K}{N \mathcal{J}_{s}} \sin \varphi \cos \theta\left(2 \cos ^{2} \theta-\mathrm{I}\right)
$$

Ces deux relations relient $\mathfrak{J}_{Y}$ et $H$ par l'intermédiaire du paramètre $\theta$; le problème est donc résolu. Lorsque le champ intérieur est faible, $\mathrm{OA}^{\prime}$ est voisin de OA : on peut alors poser $\cos \theta=\frac{\sqrt{2}}{2}+\varepsilon$ où $\varepsilon$ est très petit; en négligeant $\varepsilon^{2}$ et en éliminant $\varepsilon$ entre (21) et (22), nous obtenons

$$
\mathfrak{J}_{N}=-\left(H-\frac{N \mathscr{J}_{s}}{\sqrt{2} \cos \varphi}\right) \frac{\cos \varphi \sin \varphi}{N\left(\mathrm{I}+\frac{N \mathscr{J}_{s}^{2}}{4 K}\right)} .
$$

Nous avons vu plus haut que l'aimantation normale restait nulle jusqu'à ce que le champ $H$ atteigne la valeur $H_{r}=N \mathcal{J}_{s}: \sqrt{2} \cos \varphi$. La formule (23) nous montre qu'au-dessus, l'aimantation normale commence par être proportionnelle à $H-H_{r}$.

Il serait aussi facile de calculer maintenant la composante parallèle de l'aimantation ainsi que les proportions respectives des deux phases, mais ce serait sans intérêt. Remarquons simplement que les variations d'aimantation observées ici proviennent à la fois d'une rotation vraie de l'aimantation spontanée à l'intérieur des domaines et d'un changement dans la répartition des domaines aimantés suivant $\mathrm{OA}^{\prime}$ et $\mathrm{OB}^{\prime}$. A mesure que $H$ croît le point $\mathrm{F}$ se rapproche de $A^{\prime}$ et, à un certain moment, se confond avec lui. Tous les domaines sont alors aimantés suivant la direction $\mathrm{OA}^{\prime}$ et, dès lors, les changements d'aimantation proviennent uniquement d'une rotation réversible du vecteur $\mathrm{OA}^{\prime}$ sous l'action du champ $H$; il s'agit donc d'une aimantation selon le mode IV.

Le calcul de la composante normale dans ce dernier mode d'aimantation est classique [12] : rappelons-le très brièvement. L'énergie totale est la somme du terme d'énergie magnétocristalline

$$
-K \cos 4 \theta: 8
$$

et de l'énergie dans le champ extérieur

$$
-\boldsymbol{H} \mathfrak{J}_{s} \cos (\varphi-\theta) \text {. }
$$

Il n'y a pas à tenir compte de l'énergie due au champ démagnétisant, car il s'agit simplement ici de la rotation d'un vecteur, constant en module, suivant des directions dont le coefficient de champ démagnétisant est constant, l'énergie correspondante est donc également constante. L'énergie totale est minimum lorsque $\theta$ satisfait à la relation

$$
K \sin 4 \theta=\leadsto \mathcal{J}_{s} \sin (\varphi-\theta) .
$$

La composante normale est alors

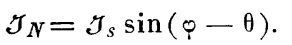

En résumé, dans un disque, la courbe de la compo- sante normale de l'aimantation en fonction du champ extérieur comporte trois régions : une première région, correspondant au mode I, dans laquelle l'aimantation normale est nulle; une deuxième région, correspondant au mode III, dans laquelle elle augmente rapidement avec le champ à partir de zéro suivant les formules (21) et (22) et enfin une dernière région, correspondant au mode IV, dans laquelle elle diminue avec le champ suivant la formule établie dans l'alinéa précédent. Nous avons comparé ces résultats théoriques aux expériences de Webster [ı3] et de Honda et Kaya [14]. Les

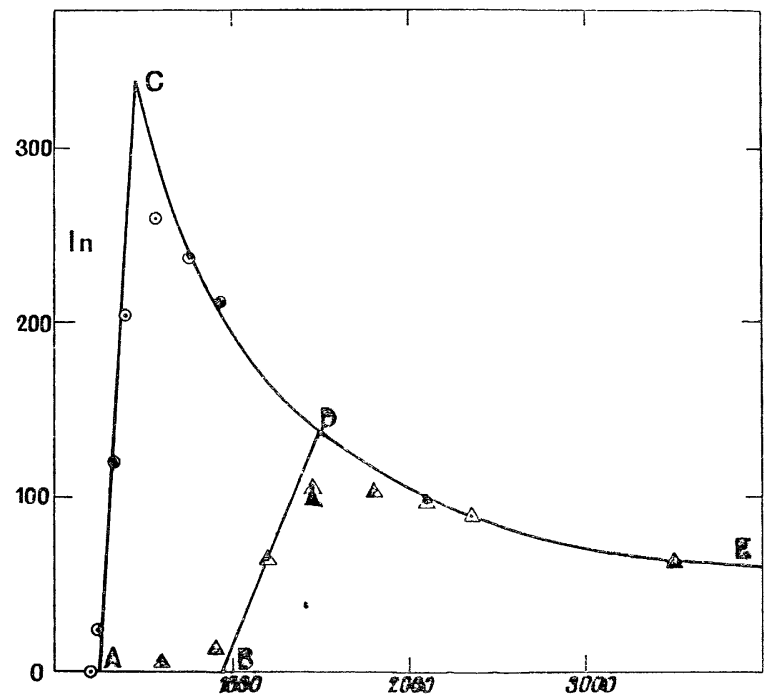

Fig. 8. - Composante normale au champ de l'aimantation, dans des disques taillés dans un des plans de base du cube. Les courbes en trait plein sont les courbes théoriques; les cercles se rapportent aux expériences de Honda et Kaya, les triangles à celles de Webster. En abscisses, on a porté le champ magnétique.

courbes de la figure 8 ont été calculées en adoptant $K=4,3 . \mathrm{IO}^{5}, \quad \check{J}_{s}=\mathrm{I} 7 \mathrm{IO}$ et en prenant, comme coefficients de champ démagnétisant, les valeurs théoriques $N=0,734$ et $N=$ o, i 90 respectivement indiquées par ces auteurs. Les portions de courbe correspondant au mode I sont représentées par les segments $\mathrm{OA}$ et $\mathrm{OB}$, celles qui correspondent au mode III par les segments $\mathrm{AC}$ et $\mathrm{BD}$ : elles dépendent de la valeur du champ démagnétisant, tandis que la portion de courbe commune CDE, correspondant au mode IV, en est indépendante. Les points expérimentaux se placent convenablement par rapport aux courbes théoriques et les écarts sont imputables tant aux impuretés et aux déformations du cristal qu'aux imperfections inévitables de la taille en ellipsoïde.

10. Les subdivisions de la courbe d'aimantation. - L'ensemble des résultats précédents 
nous prouve ainsi que, dans le cas général (orientation quelconque des axes cristallins par rapport aux axes de l'ellipsoïde), la courbe d'aimantation d'un monocristal de fer ellipsoïdal se subdivise en quatre régions correspondant aux quatre modes d'aimantation prévus dans le Tableau I du paragraphe 4; ils se succèdent dans l'ordre indiqué, au fur et à mesure que le champ extérieur croît. Aux valeurs critiques du champ correspondant au passage d'un mode à un autre, l'aimantation ne subit pas de discontinuité : la courbe présente simplement un point anguleux indiquant une discontinuité de la dérivée première $\mathrm{d} \mathscr{J}: \mathrm{d} H$. L'importance relative des différentes régions est très variable selon l'orientation des axes du cristal. Pour certaines orientations privilégiées, un ou plusieurs modes peuvent même disparaître complètement.

Chacun de ces modes est caractérisé par l'existence d'un nombre bien déterminé de phases en équilibre, $6,3,2$ ou $\mathrm{I}$, dont les volumes respectifs varient avec le champ; ces volumes sont calculables d'après les méthodes iridiquées. La direction de l'aimantation spontanée de chacune des phases varie également avec le champ, sauf dans le mode I où elle coïncide avec un axe quaternaire.

Tous ces résultats, énoncés et précisés ici pour la première fois, ont été obtenus sans rien préjuger de la forme et des dispositions relatives des domaines élémentaires : c'est ce dernier problème que nous allons maintenant étudier en prenant pour base des faits expérimentaux. Nous étudierons tout spécialement les domaines correspondants aux modes II et III, au-dessus du premier coude de la courbe d'aimantation. En effet, comme nous l'avons fait remarquer à la fin du paragraphe 5, dans le mode I, au-dessous du premier coude, les proportions relatives des six espèces de domaines élémentaires ne sont pas déterminées dans un cristal idéal bien que l'aimantation résultante le soit : ce sont des accidents minimes, tensions, inclusions, etc., qui achèveront de fixer la répartition qui pourra ainsi être très variable d'un cristal à l'autre : le polissage par exemple joue un rôle considérable et modifie profondément la structure superficielle des domaines. C'est pourquoi nous nous limiterons provisoirement $\left({ }^{(1)}\right.$ ici à l'étude de la structure des domaines correspondants aux modes II et III, entièrement déterminée par la théorie exposée plus haut et beaucoup moins sensible aux perturbations. Quant au mode IV, la question ne se pose pas puisque, par définition, ll ne possède qu'une seule espèce de domaines élémentaires.

(A suivre.)

(1) Ưn mémoire ultérieur sera consacré à l'étude du mode I.

BIBLIOGRAPHIE.

[1] Z. f. Physik, г 932,74, p. 295.

[2] Phil. Mag., 1937, 4, p. 641.

[3] Physik. Z. der Sowjetunion, i 935,8 , p. i 53.

[4] Nature, i 933,132 , p. 5 i 7 .

[5] C $f$. Honda et Kaya, Sc. Rep. Tohoku Imp. Univ. I 926,15, p. 72 г et 756 . - KAYA, Ibid., I928, 17, p. 639 .

[6] Z. f. Physik, г 933,84, p. 705 .

[7] Nature, i $933, \mathbf{1 3 2}$, p. 5i 7 .
[8] Z. f. Physik, 1931, 69, p. 78; Cf. aussi Becker, Ferromagnetismus, p. х 7 .

[9 Z. f. Physik, I933, 84, p. 705 .

[10] Z.f. Physik, 1929, 56, p. 649 .

[1 I] Akulov, Z. $f$. Physik, 1931, 69, p. 78 ; $f$. aussi Becker, Ferromagetismus, p. 117 . Nous avons reproduit ce calcul dans le paragraphe 9 .

[12] Cf. Becker, Ferromagnetismus, p. I 22.

[13] Proc. Roy. Soc. London, 1925, 107, p. 496.

[14] Sc. Rep. Tohoku Imp. Univ., 1926, 15, p. 721. 